\title{
Desarrollo y validación de un cuestionario de compatibilidad macroergonómica
}

\author{
Developing and validating a macro-ergonomic compatibility \\ questionnaire
}

\author{
Arturo Realyvásquez Vargas ${ }^{\mathrm{a}, *}$, Jorge Luis García Alcaraz ${ }^{\mathrm{a}}$ \\ y Julio Blanco Fernández ${ }^{\mathrm{b}}$ \\ a Universidad Autónoma de Ciudad Juárez, México \\ b Universidad de la Rioja, España
}

Recibido el 5 de octubre de 2015; aceptado el 14 de marzo de 2016

Disponible en Internet el 12 de mayo de 2016

\section{Resumen}

Este artículo presenta el desarrollo del Cuestionario de Compatibilidad Macroergonómica (CCM), el cual se enfoca en colectar datos sobre la aplicación de prácticas macroergonómicas respecto a los factores capital humano, organización, tareas, herramientas y tecnología, y condiciones ambientales en los sistemas de manufactura. El CCM comprende 3 secciones: datos demográficos, aplicación de prácticas macroergonómicas e importancia de estas. Las preguntas están formuladas con base en la revisión de literatura, y respondidas en escalas ordinales. La validación del CCM se realiza a través de un análisis factorial, y la opinión de los expertos. El CCM se aplicó a 188 empleados de sistemas de manufactura y 6 expertos en Ergonomía. De los empleados se obtuvo que solo 8 de las 23 variables analizadas tuvieron un valor de alfa de Cronbach $<0.7$, sin embargo, de los expertos se obtuvo que 5 de estas 8 variables fueron las más importantes dentro de su grupo, por lo cual se conservaron dentro del cuestionario.

Derechos Reservados ( 2016 Universidad Nacional Autónoma de México, Facultad de Contaduría y Administración. Este es un artículo de acceso abierto distribuido bajo los términos de la Licencia Creative Commons CC BY-NC-ND 4.0.

\section{Códigos JEL: C83; L60}

Palabras clave: Cuestionario de Compatibilidad Macroergonómica; Sistemas de manufactura; Validación del cuestionario; Elementos macroergonómicos; Prácticas macroergonómicas

\footnotetext{
* Autor para correspondencia.

Correo electrónico: al132686@alumnos.uacj.mx (A. Realyvásquez Vargas).

La revisión por pares es responsabilidad de la Universidad Nacional Autónoma de México.
} 


\begin{abstract}
This paper presents the development of a Macro-ergonomic Compatibility Questionnaire (MCQ), which focuses on collecting data about the implementation of macro-ergonomic principles which are related to human capital factors, organization, tasks, tools, technology as well as environmental conditions in manufacturing systems. The MCQ includes 3 sections: demographic data, application of macro-ergonomic principles and their importance. The questions are created according to the literature review, which are designed to be answered in ordinal scale. The MCQ validation is performed by a factorial analysis and experts' opinion. This instrument was administered to 188 manufacturing systems' employees and 6 ergonomics experts. The employees' results showed that only 8 of the 23 variables analyzed presented a value of Cronbach's alpha $<0.7$, however, experts' results presented that 5 of 8 variables were the most important in their group, therefore they were maintained in the questionnaire.

All Rights Reserved @ 2016 Universidad Nacional Autónoma de México, Facultad de Contaduría y Administración. This is an open access item distributed under the Creative Commons CC License BY-NC-ND 4.0.
\end{abstract}

JEL classification: C83; L60

Keywords: Macro-ergonomic Compatibility Questionnaire; Manufacturing systems; Questionnaire validation; Macroergonomic elements; Macroergonomic practices

\title{
Introducción
}

Ergonomía es la disciplina científica que se encarga del conocimiento teórico y fundamental del comportamiento y desempeño humano en los sistemas de interacción sociotécnica, así como de la aplicación de dicho conocimiento para el diseño de las interacciones en escenarios reales (Wilson, 2000). La Ergonomía puede traer varios beneficios para las empresas de manufactura que la aplican adecuadamente, como por ejemplo, mejorar los procesos de producción, disminuir el nivel de riesgos de trabajo y mejorar la calidad de vida laboral de los empleados, lo cual a su vez se traduce en una ventaja competitiva para la empresa (García-Acosta y Lange-Morales, 2008). La Ergonomía se divide en 2 subdisciplinas: la Microergonomía y la Macroergonomía. La Microergonomía se encarga del análisis de las interacciones hombre-máquina en contraposición a factores externos, mientras que la Macroergonomía hace hincapié en una visión más amplia de todo el diseño de sistemas de trabajo, examinando los entornos organizacionales, cultura, historia y objetivos definidos de manera amplia (Morel, Amalberti y Chauvin, 2009).

Varios cuestionarios han sido desarrollados y validados con el propósito de evaluar y mejorar el diseño del trabajo. Por ejemplo, Karasek et al. (1998) formularon el Cuestionario del Contenido de Trabajo (Job Content Questionnaire, JCQ), y Morgeson y Humphrey (2006) crearon el Cuestionario del Diseño del Trabajo (Work Design Questionnaire, WDQ). Ambos cuestionarios se enfocan en recabar información sobre las demandas, ventajas y condiciones de una tarea específica, es decir, a nivel microergonómico. Por otro lado, Carayon y Hoonakker (2004) desarrollaron el Cuestionario Organizacional Macroergonómico (Macroergonomic Organizational Questionnaire Survey, MOQS), el cual, más que ser un cuestionario, representa una metodología para desarrollar un cuestionario macroergonómico.

Algunos otros cuestionarios para evaluar y mejorar el diseño del trabajo son el Cuestionario de Diagnóstico Organizacional (Organizational Diagnosis Questionnaire, ODQ) (Preziosi, 1980) y el Cuestionario de Factores del Trabajo (Job Factors Questionnaire, JFQ) (Coluci, Alexandre 
Tabla 1

Cuestionarios para la obtención de datos de Ergonomía

\begin{tabular}{|c|c|c|c|c|}
\hline Instrumento & Autor (año) & Detalles & Enfocado a & Confiabilidad \\
\hline $\begin{array}{l}\text { Michigan Organizational } \\
\text { Assessment Questionnaire } \\
\text { (MOAQ) }\end{array}$ & $\begin{array}{l}\text { Cammann, Fichman, } \\
\text { Jenkins y Klesh } \\
(1979)\end{array}$ & $\begin{array}{l}\text { Aplicado a nivel } \\
\text { microergonómico }\end{array}$ & $\begin{array}{l}\text { Estaciones } \\
\text { de trabajo }\end{array}$ & $\alpha=0.84$ \\
\hline ODQ & Preziosi (1980) & $\begin{array}{l}35 \text { preguntas } \\
\text { Aplicado a nivel } \\
\text { macroergonómico }\end{array}$ & $\begin{array}{l}\text { Sistemas } \\
\text { de trabajo }\end{array}$ & $\mathrm{N} / \mathrm{P}$ \\
\hline JCQ & Karasek (1985) & $\begin{array}{l}49 \text { preguntas } \\
\text { Aplicado a nivel } \\
\text { microergonómico }\end{array}$ & $\begin{array}{l}\text { Estaciones } \\
\text { de trabajo }\end{array}$ & $\alpha=0.735$ \\
\hline JFQ & Coluci et al. (2009) & $\begin{array}{l}15 \text { preguntas } \\
\text { Aplicado a nivel } \\
\text { microergonómico }\end{array}$ & $\begin{array}{l}\text { Estaciones } \\
\text { de trabajo }\end{array}$ & $\kappa=0.80$ \\
\hline $\begin{array}{l}\text { Dutch Musculoskeletal } \\
\text { Questionnaire (DMQ) }\end{array}$ & $\begin{array}{l}\text { Hildebrandt, Bongers, } \\
\text { van Dijk, Kemper y } \\
\text { Dul (2001) }\end{array}$ & $\begin{array}{l}63 \text { preguntas } \\
\text { Aplicado a nivel } \\
\text { microergonómico }\end{array}$ & $\begin{array}{l}\text { Estaciones } \\
\text { de trabajo }\end{array}$ & $\alpha=0.95$ \\
\hline MOQS & $\begin{array}{l}\text { Carayon y Hoonakker } \\
\text { (2004) }\end{array}$ & $\begin{array}{l}\text { Ofrece una metodología } \\
\text { para el desarrollo e } \\
\text { implementación de } \\
\text { cuestionarios } \\
\text { macroergonómicos }\end{array}$ & $\begin{array}{l}\text { Sistemas } \\
\text { de trabajo }\end{array}$ & N/A \\
\hline WDQ & $\begin{array}{l}\text { Morgeson y } \\
\text { Humphrey (2006) }\end{array}$ & $\begin{array}{l}76 \text { preguntas } \\
\text { Aplicado a nivel } \\
\text { microergonómico }\end{array}$ & $\begin{array}{l}\text { Estaciones } \\
\text { de trabajo }\end{array}$ & $\alpha=0.87$ \\
\hline
\end{tabular}

N/A: no aplica; N/P: no proporcionado.

Fuente: Elaboración propia.

y Rosecrance, 2009). Sin embargo, ninguno de ellos recoge de manera simultánea información sobre las prácticas macroergonómicas (PM) realizadas dentro de los sistemas de manufactura en relación con el capital humano, las condiciones organizacionales, las herramientas y tecnología utilizadas por los trabajadores, y las condiciones ambientales a las cuales estos están expuestos. La tabla 1 muestra cuestionarios utilizados para obtener información sobre aspectos ergonómicos.

Se puede observar que solo 2 instrumentos (MOQS y ODQ) tienen un enfoque macroergonómico, sin embargo, de estos solo el ODQ representa un cuestionario, del cual se desconoce su confiabilidad, y únicamente recaba información sobre cuestiones organizacionales. Por otro lado el cuestionario presentado en este artículo es un cuestionario validado estadísticamente que recoge información sobre la aplicación de PM respecto a los factores de capital humano, organización, tecnología, tareas y condiciones ambientales en sistemas de manufactura.

\section{Enfoque de la investigación}

El principal propósito de esta investigación es desarrollar y validar un Cuestionario de Compatibilidad Macroergonómica (CCM) para obtener información sobre la aplicación de PM en los sistemas de manufactura. De manera más específica, el propósito del CCM es obtener información para medir la compatibilidad macroergonómica de los sistemas de manufactura, 
así como de los factores y elementos macroergonómicos que lo componen. Debido a que las PM sirven como punto de partida para medir dicha compatibilidad, se busca medir también la importancia asignada a estas prácticas para cada uno de los elementos macroergonómicos. De manera general, los 2 conceptos fundamentales de investigación en el CCM serían:

1. Obtener información sobre la aplicación de PM en las empresas y la percepción que tienen los trabajadores sobre las mismas.

2. Determinar la importancia que dan los trabajadores a la aplicación de PM respecto a un determinado elemento de su empresa.

\section{Contexto de la investigación}

El sector maquilador es clave en la industria de México, ya que incluye 5,024 establecimientos manufactureros, lo que genera un total de 2,280,504 empleos directos a nivel nacional y una facturación de 7,233.37 millones de US\$ por trimestre. El estudio aquí presentado analiza la industria maquiladora del estado de Chihuahua, uno de los 31 estados que conforman el país. El Estado de Chihuahua representa el 13.6\% de los ingresos totales de la industria maquiladora de México, empleando a 323,794 trabajadores y se encuentran establecidas 477 empresas. (InstitutoNacional-de-Estadística-Geografía-e-Informática-(INEGI) (INEGI, 2015).

Debido al entorno de trabajo industrial que se tiene en esa región, el objetivo de este artículo es presentar el desarrollo y validación del CCM, el cual se enfoca en obtener información sobre las PM efectuadas dentro de los sistemas de manufactura para cada uno de los factores macroergonómicos. La información obtenida a través del CCM podrá ser utilizada para evaluar la compatibilidad macroergonómica de los sistemas de manufactura, así como sus factores y elementos macroergonómicos.

\section{Metodología}

La metodología para la obtención del CCM comprende el desarrollo del mismo, su aplicación a empresas de la industria de manufactura de Chihuahua, México y la validación del mismo. La figura 1 muestra la secuencia de la metodología aplicada.

\section{Desarrollo del CCM}

\section{Formulación inicial de preguntas}

El desarrollo del CCM comprende 3 secciones: datos demográficos de la empresa, aplicación de PM y la importancia asignada a estas.

La sección de datos demográficos recaba información sobre género de la persona encuestada, el puesto que actualmente desempeña y antigüedad en el mismo; además del tipo de sistema de manufactura utilizado en esta y los métodos macroergonómicos que aplica.

Un paso básico en la investigación es la determinación de las variables que permiten medir la compatibilidad macroergonómica de los sistemas de manufactura y formular preguntas sobre las PM en dichas variables. Algunos autores, como Kleiner (1998, 2006, 2008), definen 4 principales variables macroergonómicas: capital humano, condiciones organizacionales, herramientas y tecnología, y condiciones ambientales. Por otro lado, el modelo de Carayon et al. (2006) incluye, además de las 4 variables definidas por Kleiner $(1998,2006,2008)$, la variable tareas. Estas variables son conocidas como factores macroergonómicos y son divididos en variables más simples 


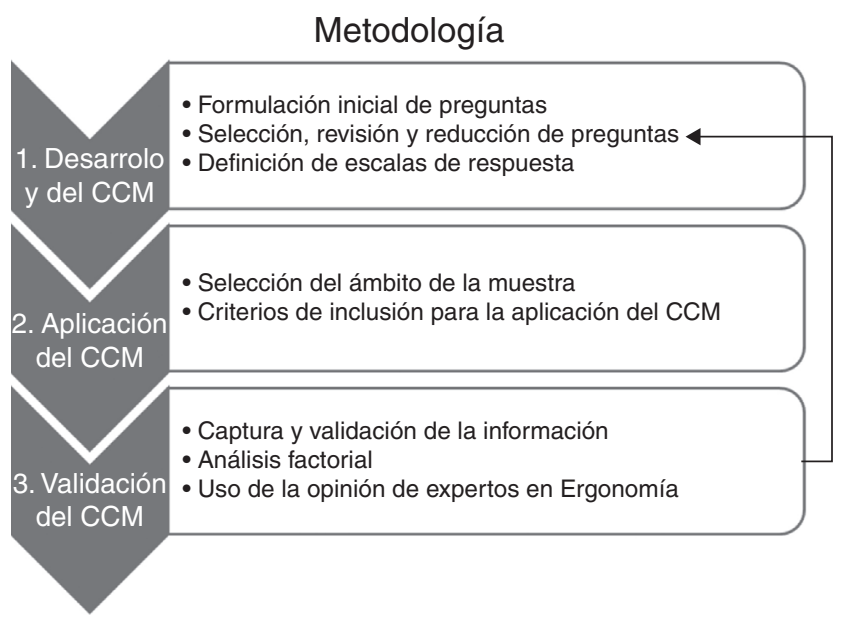

Figura 1. Metodología para el desarrollo y validación del CCM.

Fuente: Elaboración propia.

conocidas como elementos macroergonómicos, los cuales son tomados como base y adaptados para la formulación de preguntas sobre las PM en sistemas de manufactura.

La tabla 2 muestra la revisión de literatura para cada factor macroergonómico y sus correspondientes elementos.

\section{Revisión, selección y reducción de preguntas en el CCM}

A partir de 150 preguntas iniciales se realiza una revisión de su contenido con la finalidad de eliminar aquellas que tengan significados similares. La revisión se lleva a cabo en colaboración con personal experto en Ergonomía y/o personal con experiencia en la elaboración de cuestionarios.

\section{Definición de escalas de respuesta}

Para las respuestas del CCM se utiliza una escala tipo Likert difusa de 5 pasos, la cual ha sido aplicada de manera efectiva en otros estudios recientes y similares (García, Maldonado, Alvarado y Rivera, 2014; Glover, Farris, van Aken y Doolen, 2011; Li, 2013; Likert, 1932), la cual permite medir el grado en el cual los sistemas de manufactura aplican las PM. Los pasos de dicha escala son: 1) Totalmente en desacuerdo, 2) Muy en desacuerdo, 3) Ni de acuerdo ni en desacuerdo, 4) Muy de acuerdo, 5) Totalmente en desacuerdo. Esta escala se utiliza para medir el grado de aplicación de PM en los sistemas de manufactura.

Para las preguntas sobre la importancia que asignan los empleados de los sistemas de manufactura y el personal experto en ergonomía a la realización de PM en cada uno de los elementos macroergonómicos se utiliza la escala difusa (Celik, Kahraman, Cebi y Er, 2009), la cual también comprende 5 pasos: 1) Muy bajo, 2) Bajo, 3) Medio, 4) Alto, 5) Muy alto.

\section{Aplicación del CCM}

\section{Selección del ámbito de la muestra}

El personal encuestado comprende 2 grupos: 1) mandos medios y superiores de sistemas de manufactura, y 2) personal experto en Ergonomía. Del primer grupo se obtiene 
Tabla 2

Revisión de literatura para los elementos macroergonómicos

Factores y sus elementos
macroergonómicos

\section{Capital humano}

Características físicas

Motivación y necesidades

Educación, habilidades y conocimientos

Características psicológicas

\section{Organización}

Estilos de supervisión y

administración

Horarios de trabajo

Cultura organizacional y cultura de seguridad del trabajador

Coordinación, colaboración y comunicación Evaluación del desempeño, recompensas e incentivos Trabajo en equipo

Relaciones sociales

\section{Herramientas y tecnología \\ Tecnología de la información}

Tecnología de manufactura avanzada
(Humphrey, Nahrgang y Morgeson, 2007), (Zink, 2014), (Zhao, McCoy, Kleiner, Smith-Jackson y Liu, 2015), (Horst, Broday, Bondarik y de Paula Xavier, 2015) (Preziosi, 1980), (Morgeson y Humphrey, 2003), (Nakata et al., 2004), (Morgeson y Humphrey, 2006), (Humphrey et al., 2007), (Zink, 2014), (Zhao et al., 2015) (Preziosi, 1980), (Morgeson y Humphrey, 2003), (Nakata et al., 2004), (Morgeson y Humphrey, 2006), (Humphrey et al., 2007), (Zink, 2014), (Zhao et al., 2015)

(Karasek et al., 1998), (Nakata et al., 2004), (Zink, 2014), (Zhao et al., 2015)

(Karasek et al., 1998), (Morgeson y Humphrey, 2003), (Nakata et al., 2004), (Morgeson y Humphrey, 2006), (Zink, 2014), (Realyvásquez, Maldonado-Macías, García-Alcaraz y Blanco-Fernández, 2015), (Horst et al., 2015)

(Anderson, Binder y Krause, 2002), (Lipscomb, Trinkoff, Geiger-Brown y Brady, 2002), (Trinkoff, Le, Geiger-Brown y Lipscomb, 2007), (̊kerstedt, Fredlund, Gillberg y Jansson, 2002), (Beckers et al., 2004), (Morgeson y Humphrey, 2003), (Morgeson y Humphrey, 2006), (Realyvásquez et al., 2015), (Bolis y Sznelwar, 2015) (Preziosi, 1980), (Choudhry, Fang y Mohamed, 2007), (Giunta, Nifo y Scalera, 2012), (Realyvásquez et al., 2015), (Horst et al., 2015)

(Preziosi, 1980), (Nakata et al., 2004), (Realyvásquez et al., 2015), (Balbinotti y Paupitz, 2015)

(Preziosi, 1980), (Roberts, 2003), (Zhao et al., 2015), (Horst et al., 2015)

(Preziosi, 1980), (Morgeson y Humphrey, 2003), (Nakata et al., 2004), (Morgeson y Humphrey, 2006), (Humphrey et al., 2007), (Oldham y Cummings, 1996), (Axtell et al., 2000), (Detert y Burris, 2007), (Realyvásquez et al., 2015), (Horst et al., 2015)

(Preziosi, 1980), (Morgeson y Humphrey, 2003), (Nakata et al., 2004), (Morgeson y Humphrey, 2006), (Realyvásquez et al., 2015), (Horst et al., 2015)

(Morgeson y Humphrey, 2003), (Morgeson y Humphrey, 2006), (Mital et al., 1999), (Pennathur y Mital, 2003), (Mital y Pennathur, 2004), (Golshan y Tafazoli, 2014), (Di Pietro, Pantano y di Virgilio, 2014), (Morosan y DeFranco, 2014), (Zhu y Chang, 2014), (Yilmaz y Bayraktar, 2014), (Valdez y Zayas-Cabánb, 2015), (Yang y Rivera, 2015) (Morgeson y Humphrey, 2003), (Morgeson y Humphrey, 2006), (Maldonado et al., 2009), (Maldonado, García, Alvarado y Balderrama, 2013) 
Tabla 2 (Continuación)

\begin{tabular}{|c|c|}
\hline $\begin{array}{l}\text { Factores y sus elementos } \\
\text { macroergonómicos }\end{array}$ & Autores \\
\hline $\begin{array}{l}\text { Características de factores } \\
\text { humanos en tecnologías y } \\
\text { herramientas }\end{array}$ & $\begin{array}{l}\text { (Sen y Sahu, 1996), (Jung y Hallbeck, 2005), (You, Kumar, } \\
\text { Young, Veluswamy y Malzahn, 2005), (Zhao et al., 2015), } \\
\text { (Horst et al., 2015), (Yang y Rivera, 2015) }\end{array}$ \\
\hline \multicolumn{2}{|l|}{ Tareas } \\
\hline Variedad de tareas & $\begin{array}{l}\text { (Morgeson y Humphrey, 2003), (Morgeson y Humphrey, } \\
\text { 2006), (Pavlovic-Veselinovic, Hedge y Veselinovic, 2016) }\end{array}$ \\
\hline $\begin{array}{l}\text { Demanda de trabajo (carga de } \\
\text { trabajo, atención requerida, etc.) }\end{array}$ & $\begin{array}{l}\text { (Van der Doef y Maes, 1999), (Häusser, Mojzisch, Niesel y } \\
\text { Schulz-Hardt, 2010), (Zink, 2014) }\end{array}$ \\
\hline $\begin{array}{l}\text { Contenido de trabajo, desafío y } \\
\text { utilización de habilidades }\end{array}$ & $\begin{array}{l}\text { (Karasek et al., 1998), (Zink, 2014), (Zhao et al., 2015), } \\
\text { (Balbinotti y Paupitz, 2015) }\end{array}$ \\
\hline $\begin{array}{l}\text { Autonomía, control de trabajo y } \\
\text { participación }\end{array}$ & $\begin{array}{l}\text { (Van der Doef y Maes, 1999), (Thompson y Prottas, 2006), } \\
\text { (Zink, 2014), (Zhao et al., 2015), (Robertsona, Huanga y } \\
\text { Leea, 2015) }\end{array}$ \\
\hline \multicolumn{2}{|l|}{ Condiciones ambientales } \\
\hline Ruido & $\begin{array}{l}\text { (Nakata et al., 2004), (Morgeson y Humphrey, 2003), } \\
\text { (Morgeson y Humphrey, 2006), (Humphrey et al., 2007), } \\
\text { (Zink, 2014), (Wilson y Sharples, 2015) }\end{array}$ \\
\hline Diseño de la estación de trabajo & $\begin{array}{l}\text { (Morgeson y Humphrey, 2003), (Morgeson y Humphrey, } \\
\text { 2006), (Oyewole, Haight y Freivalds, 2010), (Öztürk y Esin, } \\
\text { 2011), (Robertsona et al., 2015), (Bolis y Sznelwar, 2015) }\end{array}$ \\
\hline Iluminación & $\begin{array}{l}\text { (Nakata et al., 2004), (Zhao et al., 2015), (Reinhold y Tint, } \\
\text { 2015) }\end{array}$ \\
\hline $\begin{array}{l}\text { Temperatura, humedad y calidad } \\
\text { del aire }\end{array}$ & $\begin{array}{l}\text { (Nakata et al., 2004), (Morgeson y Humphrey, 2003), } \\
\text { (Morgeson y Humphrey, 2006), (Humphrey et al., 2007), } \\
\text { (Seim y Broberg, 2010), (Zhao et al., 2015) }\end{array}$ \\
\hline Distribución & $\begin{array}{l}\text { (Morgeson y Humphrey, 2003), (Morgeson y Humphrey, } \\
\text { 2006), (Seim y Broberg, 2010), (Bolis y Sznelwar, 2015) }\end{array}$ \\
\hline
\end{tabular}

Fuente: Elaboración propia.

información sobre las PM aplicadas en los sistemas de manufactura, mientras que el segundo grupo permite acceder a información relacionada con la importancia de las PM para cada uno de los elementos macroergonómicos, por lo que para este último grupo solo aplica la sección 3 del CCM.

La información de contacto para los miembros del primer grupo se ha obtenido de las base de datos del INEGI, y de la Asociación de Maquiladoras, A. C. (AMAC), actualmente denominada INDEX. Estas listas permiten el acceso a listas de registro de empresas del sector industrial en Chihuahua, México.

\section{Criterios de inclusión para la aplicación del CCM}

Como se ha indicado en el párrafo anterior, el cuestionario se aplica a mandos medios y superiores de sistemas de manufactura. Para los empleados de sistemas de manufactura, la elección de los encuestados entre los mandos medios y superiores se debe a que estos tienen una visión más general del sistema de manufactura en el que trabajan, sus operaciones, deficiencias y oportunidades de mejora. El personal encuestado incluye ingenieros, supervisores, gerentes y jefes de grupo. 
Para el personal experto en Ergonomía se aplica un cuestionario que únicamente incluye la tercera sección del CCM. A continuación se listan los principios de inclusión de los expertos en Ergonomía:

1. Poseer experiencia en la industria de manufactura.

2. Estar en posesión de una certificación en Ergonomía.

3. Pertenecer, o haber pertenecido a organizaciones nacionales y/o internacionales de Ergonomía.

4. Tener publicaciones en revistas y/o congresos.

5. Contar con una experiencia mínima de 10 años en el área de Ergonomía.

6. Poseer experiencia en Salud Ocupacional.

7. Contar con un título de posgrado relacionado con Ergonomía.

\section{Validación del CCM}

\section{Captura y validación de la información}

Los datos obtenidos de las empresas de manufactura son capturados en el software Pasw Statistic 21®. Antes del proceso de validación, se ha realizado una depuración de los datos sustituyendo los valores perdidos por la mediana, esto debido a que los datos se encuentran en una escala ordinal (en una escala tipo Likert) (García et al., 2014; Hair, Tatham, Anderson y Black, 2006), pero también se han estandarizado los ítems o variables para identificar posibles valores extremos, para lo cual se realizaron diagramas de caja y un proceso de estandarización, donde se considera valor extremo a una observación que tiene un valor absoluto estandarizado mayor a 4. Esos valores extremos fueron sustituidos con la mediana de los datos también.

La validación del cuestionario se ha realizado en 2 etapas, la primera validación se ha obtenido mediante el índice alfa de Cronbach para cada dimensión (elemento macroergonómco), para los cuales se busca que existan valores superiores a 0.7 , ya que ese es el valor de corte recomendado por varios autores (Cronbach, 1951; Fornell y Larcker, 1981; García et al., 2014; Nunnally, 1978; Nunnally y Bernstein, 2005). Después, se ha realizado un análisis factorial a cada uno de los elementos macroergonómicos, donde se aplica la prueba de esfericidad de Bartlett y la prueba de Kaiser-Meyer-Olkin para determinar la viabilidad del mismo (Schulze, Hilger y Engelberg, 2015). La rotación elegida fue varimax por ofrecer una mayor extracción de la varianza y asignarla a solamente un factor (Wang, Liu y Tu, 2005), pero además, con la finalidad de minimizar el número de ítems en cada una de las variables analizadas, se ha hecho uso de aquellas que tuvieran una comunalidad mayor a 0.5 (Kamboj et al., 2014).

\section{Opinión de expertos en ergonomía}

Al igual que los datos proporcionados por las empresas, los datos ofrecidos por los expertos también son capturados en el software Pasw Statistic 21®, con la finalidad de obtener la mediana de la importancia en una escala de 1 a 5 , proporcionada para cada uno de los elementos macroergonómicos.

La opinión del personal experto en Ergonomía es comparada con los resultados obtenidos en el análisis factorial, con la finalidad de determinar si aquellos elementos macroergonómicos que tengan un valor de alfa de Cronbach menor a 0.7 son realmente importantes para medir la compatibilidad macroergonómica según los expertos, y así justificar su inclusión en el CCM. Como se observa en la figura 1, se retorna a la etapa 1 de la Metodología, al paso selección, revisión y reducción de preguntas, para definir la cantidad final de estas. 
Tabla 3

Cantidad de preguntas para cada factor macroergonómico en el CCM

\begin{tabular}{lllll}
\hline Capital humano & Organización & Herramientas y tecnología & Tareas & Condiciones ambientales \\
\hline 14 & 30 & 13 & 16 & 19 \\
\hline
\end{tabular}

Fuente: Elaboración propia.

\section{Resultados y discusión}

\section{Desarrollo del CCM}

De las 150 preguntas originales, se eliminaron 30,23 de las cuales correspondieron a una sección llamada frecuencia, la cual se fusionó con la sección de importancia, debido a que, según los revisores, cuanto más frecuente se aplique una PM, mayor es su importancia. Las otras 7 preguntas se eliminaron debido a que tenían significados similares con otras. Al final el CCM se desarrolló dividido en 3 secciones y comprendiendo un total de 120 preguntas divididas de la siguiente manera:

1) Sección 1: 6 preguntas para la sección de datos demográficos.

2) Sección 2: 92 preguntas para la aplicación de PM.

3) Sección 3: 22 preguntas para la asignación de importancia de las PM.

A su vez, las 92 preguntas de la sección 2 quedan divididas en los 5 factores macroergonómicos, tal como se indica en la tabla 3.

El CCM está estructurado de manera que permite obtener información detallada respecto a la aplicación de PM en cada uno de los factores macroergonómicos y sus respectivos elementos. La cantidad de 120 preguntas resulta relativamente muy alta en comparación con otros cuestionarios, esto debido a la cantidad de información que el CCM pretende recabar. Sin embargo, el CCM tiene la característica de ser flexible en cuanto a que puede ser contestado en diferentes sesiones de acuerdo a la disponibilidad de tiempo del encuestado.

\section{Aplicación del CCM}

El CCM se aplica a 188 empleados con cargos de mandos medios y superiores en empresas de manufactura de Chihuahua, México. La tabla 4 muestra la distribución de frecuencias y los porcentajes de aplicación de las encuestas del CCM.

Tabla 4

Puestos de trabajo y su frecuencia de aplicación del CCM

\begin{tabular}{llc}
\hline Puesto & Frecuencia & Porcentaje \\
\hline Gerente & 19 & $10.11 \%$ \\
Supervisor & 90 & $47.87 \%$ \\
Jefe de grupo & 51 & $27.13 \%$ \\
Administrativo & 28 & $14.89 \%$ \\
\hline
\end{tabular}

Fuente: Elaboración propia. 
Se puede observar que la mayoría de los participantes (75\%) son jefes de grupo y supervisores, los cuales tienen más interacción con la tecnología y las tareas incluidas dentro de los procesos de manufactura dentro del grupo de encuestados. Esto incrementa la credibilidad en cuanto a la aplicación de PM en las instalaciones del proceso de manufactura. Por otro lado, la cantidad de encuestados en puestos gerenciales y administrativos (25\%) representa un complemento a la aplicación de PM en los procesos de manufactura, ya que esto proporcionó información sobre las PM aplicadas principalmente en tareas de oficina.

En el caso de los expertos en Ergonomía se cuenta con la opinión de 6 profesionales del área. Los expertos cumplen ampliamente los requisitos de inclusión, tales como una amplia experiencia profesional en la industria maquiladora y poseer certificaciones en Ergonomía. También es importante señalar que prácticamente la totalidad de ellos son miembros de organizaciones internaciones o nacionales de Ergonomía, han presentado ponencias en congresos científicos o son autores de artículos de investigación en el ámbito ergonómico.

El cumplimiento de todas las características por la mayoría de los expertos garantiza una mayor confiabilidad de los resultados de la importancia de las PM y en la validación final del CCM.

\section{Validación del CCM}

En la tabla 5 se ilustra el análisis estadístico para la validación del cuestionario, en el que se muestra que la mayoría de los elementos macroergonómicos, con sus correspondientes preguntas, tienen un valor de alfa de Cronbach mayor a 0.7. Sin embargo, se puede apreciar que para algunos elementos esta condición no se cumple. En la tabla 5 también se muestra cada elemento macroergonómico con su correspondiente cantidad de preguntas formuladas, y la mediana de la importancia asignada por expertos (en una escala de 1 a 5), agrupados por factores y ordenados de manera descendente de acuerdo a la importancia dada.

Un ordenamiento general con base en la importancia dio como resultado que los expertos en Ergonomía consideran más importantes elementos como la variedad de tareas que realizan los trabajadores, la tecnología de la información y la tecnología de manufactura avanzada utilizada dentro de la empresa, el diseño de la estación de trabajo, y el ruido al que están expuestos los empleados. Mientras que los menos importantes son las relaciones sociales, el trabajo en equipo, las características psicológicas de los empleados, la evaluación del desempeño, recompensas e incentivos hacia los trabajadores, y la coordinación, colaboración y comunicación.

Algunos elementos como educación, habilidades y conocimiento, estilos de supervisión y administración, horarios de trabajo, tecnología de manufactura avanzada y variedad de tareas tienen un valor alfa de Cronbach menor a 0.7 . Por lo cual, en primera instancia deben ser eliminados del cuestionario, ya que no se tiene la suficiente confiabilidad de que explican la compatibilidad macroergonómica. Sin embargo, al comparar la segunda y tercera columna de la tabla 5 se puede ver que estos elementos tienen los valores más altos en cuanto a la importancia asignada por expertos. Por esta razón se decide conservarlos como elementos macroergonómicos dentro del CCM, aunque la estructura y legibilidad de las preguntas debe ser mejorada. Por lo anterior, se puede decir que el CCM es plenamente validado.

Al hacer un ordenamiento por grupos, tomando en cuenta la cantidad de preguntas para cada elemento, dio como resultado que, por lo general, a mayor cantidad de preguntas se obtenía un mayor valor de alfa de Cronbach. Una excepción fue el elemento de relaciones sociales, para el cual, con 6 preguntas (segundo en su grupo), se obtuvo un valor alfa de Cronbach igual a 
Tabla 5

Validación e importancia de los elementos macroergonómicos

\begin{tabular}{|c|c|c|c|}
\hline Elementos macroergonómicos & $\begin{array}{l}\text { Número de } \\
\text { preguntas }\end{array}$ & $\begin{array}{l}\text { Alfa de } \\
\text { Cronbach }\end{array}$ & $\begin{array}{l}\text { Importancia } \\
\text { dada por } \\
\text { expertos }\end{array}$ \\
\hline \multicolumn{4}{|l|}{ Capital humano } \\
\hline Características físicas & 3 & 0.702 & 4.20 \\
\hline Motivación y necesidades & 4 & 0.746 & 3.60 \\
\hline Educación, habilidades y conocimiento & 2 & 0.444 & 3.60 \\
\hline Características psicológicas & 5 & 0.877 & 3.33 \\
\hline \multicolumn{4}{|l|}{ Organización } \\
\hline Estilos de supervisión y administración & 2 & 0.569 & 4.20 \\
\hline Horarios de trabajo & 3 & 0.287 & 4.20 \\
\hline $\begin{array}{l}\text { Cultura organizacional y cultura de } \\
\text { seguridad del trabajador }\end{array}$ & 7 & 0.803 & 4.0 \\
\hline $\begin{array}{l}\text { Coordinación, colaboración y } \\
\text { comunicación }\end{array}$ & 4 & 0.710 & 3.60 \\
\hline $\begin{array}{l}\text { Evaluación del desempeño, recompensas e } \\
\text { incentivos }\end{array}$ & 4 & 0.731 & 3.50 \\
\hline Trabajo en equipo & 4 & 0.779 & 3.33 \\
\hline Relaciones sociales & 6 & 0.669 & 3.20 \\
\hline \multicolumn{4}{|l|}{ Herramientas y tecnología } \\
\hline Tecnología de la información & 7 & 0.768 & 4.40 \\
\hline Tecnología de manufactura avanzada & 4 & 0.650 & 4.40 \\
\hline $\begin{array}{l}\text { Características de factores humanos en } \\
\text { tecnologías y herramientas }\end{array}$ & 2 & 0.534 & 4.00 \\
\hline \multicolumn{4}{|l|}{ Tareas } \\
\hline Variedad de tareas & 3 & 0.449 & 4.50 \\
\hline $\begin{array}{l}\text { Demanda de trabajo (carga de trabajo, } \\
\text { atención requerida, etc.) }\end{array}$ & 6 & 0.716 & 4.33 \\
\hline $\begin{array}{l}\text { Contenido de trabajo, desafío y utilización } \\
\text { de habilidades }\end{array}$ & 2 & 0.670 & 4.20 \\
\hline $\begin{array}{l}\text { Autonomía, control de trabajo y } \\
\text { participación }\end{array}$ & 5 & 0.752 & 3.75 \\
\hline \multicolumn{4}{|l|}{ Condiciones ambientales } \\
\hline Ruido & 3 & 0.757 & 4.40 \\
\hline Diseño de la estación de trabajo & 4 & 0.734 & 4.40 \\
\hline Iluminación & 2 & 0.706 & 4.40 \\
\hline Temperatura, humedad y calidad del aire & 5 & 0.761 & 4.20 \\
\hline Distribución & 5 & 0.840 & 4.00 \\
\hline
\end{tabular}

Fuente: Elaboración propia.

0.669, que es menor a 0.7. En el anexo I se muestra el cuestionario con todas las preguntas de las secciones 1 y 2 . La sección 3 fue excluida, ya que solo recoge información sobre la importancia de aplicación de PM para cada uno de los elementos macroergonómicos.

\section{Conclusiones y recomendaciones}

Se ha desarrollado un CCM, el cual se diferencia de otros cuestionarios macroergonómicos en que contiene secciones que permiten recolectar información para tener una visión más clara, 
detallada, y a la vez general de la aplicación e importancia de PM en las empresas de manufactura. Esto es debido a que ofrece una cobertura detallada de cada elemento macroergonómico a través de las preguntas, y una cobertura general al incluir en un solo instrumento los 5 factores macroergonómicos más mencionados en la literatura. Por esto, y por los resultados obtenidos en la validación del CCM, se concluye que este es efectivo y eficiente, conteniendo las siguientes ventajas:

- Representa una herramienta y un avance en uno de los mayores desafíos que enfrentan actualmente los investigadores en el área de Ergonomía: el de crear un modelo matemático que permita medir la compatibilidad de sistemas de trabajo de manera amplia y detallada.

- Permite recabar datos para realizar análisis estadísticos tanto a nivel empresa como a nivel sector.

- Facilita la detección de elementos y factores macroergonómicos que requieren mayor nivel de aplicación de PM para así mejorar la sustentabilidad de los sistemas de trabajo.

- Permite monitorizar el avance en la aplicación de PM y comparar resultados a través del tiempo.

En un sentido académico, el CCM representa un nuevo instrumento que puede ser aplicado para evaluar la compatibilidad macroergonómica de diferentes sectores de trabajo (manufactura, salud, educación, etcétera) realizando las adaptaciones necesarias.

Como recomendaciones de futuro, los autores de este artículo consideran que es importante realizar estudios e investigaciones en cuanto a:

- Incrementar la cantidad de preguntas en aquellos elementos macroergonómicos para los cuales se obtuvo un valor alfa de Cronbach menor a 0.7 .

- La aplicación del CCM a más empresas dentro de México, EE. UU. y países de otras regiones, esto con la finalidad de incrementar la validez del CCM y el conocimiento de la aplicación de PM.

- El desarrollo de un programa informático que facilite la aplicación de CCM y el procesamiento de los datos.

- El desarrollo de un índice que permita medir la compatibilidad macroergonómica de los sistemas de manufactura a partir de la aplicación de PM, y la importancia asignada a estas por las empresas y por expertos en Ergonomía.

- Difundir y aplicar el CCM dentro de instituciones académicas con la finalidad de extender su aplicación a otros sectores industriales.

\section{Agradecimientos}

Los autores de esta investigación quieren agradecer a las empresas del sector maquilador del Estado de Chihuahua, México, la aportación de su valiosa información, lo mismo que a los expertos en Ergonomía por ayudar a definir los niveles de importancia de la aplicación de PM. De igual forma se agradece al Consejo Nacional de Ciencia y Tecnología (CONACYT) su valioso apoyo económico para la investigación. 


\section{Anexo I. Cuestionario de compatibilidad macroergonómica para sistemas de manufactura}

\section{SECCIÓN I. DATOS DEMOGRÁFICOS}

Esta primera parte solicita que proporcione datos generales de la empresa para conocer sus características sobre el personal de trabajo y sistema de manufactura.

1. Nombre de la empresa nombre de la empresa se utiliza sólo para fines de estudio, no se dará a conocer.

2. Género de la persona que responde la encuesta: Masculino

3. Puesto que actualmente desempeña

4. Antigüedad en la empresa

5. Tipo de sistema de manufactura que maneja su empresa

Celular

Flexible

Híbrido

Otro (especifique)

6. Indique cuáles de los siguientes los Programas de Ergonomía aplica dentro del sistema de manufactura de su organización

Ergonomía Participativa

Grupo focal

Análisis y diseño macroergonómico (Macroergonomic analysis and design, MEAD)

Análisis macroergonómico de la estructura (Macroergonomic Analysis of Structure. MAS)

Estudio de laboratorio (Simular un caso en laboratorio)

Experimento de campo

Cuestionario

Entrevista

Métodos microergonómicos (Análisis postural con REBA, RULA, NIOSH, OCRA,

Suzanne Rodgers)

Femenino

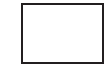

Otro (especifique): 


\section{SECCIÓN II. NIVEL DE APLICACIÓN DE PRÁCTICAS MACROERGONÓMICAS}

\section{Instrucciones}

Para contestar el cuestionario utilice la plantilla que se expone a continuación y un bolígrafo. Favor de contestar todas y cada una de las preguntas. Indique el nivel en el que está usted de acuerdo con la aplicación de las siguientes prácticas macroergonómicas.

\begin{tabular}{|c|c|c|c|c|c|}
\hline \multicolumn{6}{|c|}{ Evaluación de aspectos humanos } \\
\hline \multicolumn{6}{|l|}{ En su empresa: } \\
\hline & $\begin{array}{l}\text { otalmente } \\
\quad \text { en } \\
\text { esacuerdo }\end{array}$ & $\begin{array}{c}\text { Muy en } \\
\text { desacuerdo }\end{array}$ & $\begin{array}{l}\text { Ni de } \\
\text { acuerdo ni } \\
\quad \text { en } \\
\text { desacuerdo }\end{array}$ & $\begin{array}{l}\text { Muy de } \\
\text { acuerdo }\end{array}$ & $\begin{array}{l}\text { Totalmente } \\
\quad \text { de } \\
\text { acuerdo }\end{array}$ \\
\hline $\begin{array}{l}\text { 1.1a Se hacen contrataciones/asignaciones de tarea } \\
\text { con base en la educación, habilidades y el } \\
\text { conocimiento de los trabajadores }\end{array}$ & 1 & 2 & 3 & 4 & 5 \\
\hline $\begin{array}{l}\text { 1.1b Se promueve la educación, la adquisición de } \\
\text { habilidades y conocimiento por parte de los } \\
\text { trabajadores }\end{array}$ & 1 & 2 & 3 & 4 & 5 \\
\hline $\begin{array}{l}\text { 1.2a Se toman en consideración la motivación y las } \\
\text { necesidades de los trabajadores }\end{array}$ & 1 & 2 & 3 & 4 & 5 \\
\hline $\begin{array}{l}1.2 \mathrm{~b} \text { Se motiva a los trabajadores a realizar su } \\
\text { trabajo mediante la solución de problemas }\end{array}$ & 1 & 2 & 3 & 4 & 5 \\
\hline $\begin{array}{l}1.2 \mathrm{c} \text { Se le brinda ayuda laboral al trabajador que lo } \\
\text { necesita }\end{array}$ & 1 & 2 & 3 & 4 & 5 \\
\hline $\begin{array}{l}1.2 \mathrm{~d} \text { Existen oportunidades de promoción y } \\
\text { crecimiento laboral }\end{array}$ & 1 & 2 & 3 & 4 & 5 \\
\hline $\begin{array}{l}\text { 1.3a Se consideran las características físicas de los } \\
\text { trabajadores para la asignación de tareas }\end{array}$ & 1 & 2 & 3 & 4 & 5 \\
\hline $\begin{array}{l}\text { 1.3b Se presta atención al trabajador cuando } \\
\text { presenta molestias físicas }\end{array}$ & 1 & 2 & 3 & 4 & 5 \\
\hline $\begin{array}{l}\text { 1.3c Se estudian/analizan las causas de molestias } \\
\text { físicas en los trabajadores }\end{array}$ & 1 & 2 & 3 & 4 & 5 \\
\hline $\begin{array}{l}\text { 1.4a Se consideran las características psicológicas } \\
\text { de los trabajadores para la asignación de tareas } \\
\text { 1.4b Se presta atención al trabajador cuando }\end{array}$ & s. & 2 & 3 & 4 & 5 \\
\hline $\begin{array}{l}\text { presenta malestares psicológicos como estrés } \\
\text { mental, depresión, etc. }\end{array}$ & 1 & 2 & 3 & 4 & 5 \\
\hline $\begin{array}{l}\text { 1.4c Se estudian/analizan las causas de malestares } \\
\text { psicológicos en los trabajadores }\end{array}$ & S & 2 & 3 & 4 & 5 \\
\hline $\begin{array}{l}\text { los trabajadores no presenten malestares } \\
\text { psicológicos: estrés mental, depresión, entre otras. } \\
1.4 \text { e Las tareas están diseñadas con el fin de que }\end{array}$ & (1) & 2 & 3 & 4 & 5 \\
\hline $\begin{array}{l}\text { los trabajadores se sientan satisfechos con su } \\
\text { trabajo }\end{array}$ & 1 & 2 & 3 & 4 & 5 \\
\hline \multicolumn{6}{|c|}{ Evaluación de condiciones administrativas de la organización } \\
\hline \multicolumn{6}{|l|}{ En su empresa: } \\
\hline \multicolumn{2}{|c|}{$\begin{array}{l}\text { Totalmente } \\
\text { en } \\
\text { desacuerdo }\end{array}$} & $\begin{array}{c}\text { Muy en } \\
\text { desacuerdo }\end{array}$ & $\begin{array}{c}\text { Ni de } \\
\text { acuerdo ni } \\
\text { en } \\
\text { desacuerdo }\end{array}$ & $\begin{array}{l}\text { Muy de } \\
\text { acuerdo }\end{array}$ & $\begin{array}{c}\text { Totalmente } \\
\text { de } \\
\text { acuerdo }\end{array}$ \\
\hline 2.1a Los trabajadores realizan sus tareas en equipo & 01 & 2 & 3 & 4 & 5 \\
\hline
\end{tabular}




\begin{tabular}{|c|c|c|c|c|c|}
\hline \multicolumn{6}{|c|}{ Evaluación de condiciones administrativas de la organización } \\
\hline \multicolumn{6}{|l|}{ En su empresa: } \\
\hline & $\begin{array}{l}\text { Totalmente } \\
\text { en } \\
\text { desacuerdo }\end{array}$ & $\begin{array}{l}\text { Muy en } \\
\text { desacuerdo }\end{array}$ & $\begin{array}{c}\mathrm{Ni} \text { de } \\
\text { acuerdo ni } \\
\text { en } \\
\text { desacuerdo }\end{array}$ & $\begin{array}{l}\text { Muy de } \\
\text { acuerdo }\end{array}$ & $\begin{array}{l}\text { Totalmente } \\
\text { de } \\
\text { acuerdo }\end{array}$ \\
\hline $\begin{array}{l}\text { 2.1b Se brinda apoyo por parte de los compañeros } \\
\text { de trabajo cuando un trabajador manifiesta dudas o } \\
\text { dificultades al momento de realizar una tarea }\end{array}$ & 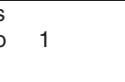 & 2 & 3 & 4 & 5 \\
\hline $\begin{array}{l}\text { 2.1c Se respetan y toman en cuenta las opiniones, } \\
\text { ideas y sugerencias de todos los trabajadores } \\
\text { 2.1d Los trabajadores reciben retroalimentación }\end{array}$ & , & 2 & 3 & 4 & 5 \\
\hline $\begin{array}{l}\text { sobre su desempeño por parte de los superiores y } \\
\text { compañeros }\end{array}$ & 1 & 2 & 3 & 4 & 5 \\
\hline $\begin{array}{l}\text { 2.2a Los trabajadores se esfuerzan por mantener } \\
\text { una buena coordinación, colaboración y } \\
\text { comunicación }\end{array}$ & 1 & 2 & 3 & 4 & 5 \\
\hline $\begin{array}{l}\text { 2.2b Cualquier trabajador se puede dirigir con otro } \\
\text { trabajador sin importar el nivel jerárquico }\end{array}$ & 1 & 2 & 3 & 4 & 5 \\
\hline $\begin{array}{l}\text { 2.2c Se da retroalimentación a los trabajadores } \\
\text { para mantener una buena comunicación a nivel } \\
\text { organizacional }\end{array}$ & 1 & 2 & 3 & 4 & 5 \\
\hline $\begin{array}{l}\text { 2.2d Se utilizan diferentes formas de } \\
\text { comunicación: escrita, oral, auditiva, visual }\end{array}$ & 1 & 2 & 3 & 4 & 5 \\
\hline $\begin{array}{l}\text { 2.3a Se transmiten los principios y valores de la } \\
\text { empresa hacia sus trabajadores de manera clara } \\
\text { desde el inicio }\end{array}$ & 1 & 2 & 3 & 4 & 5 \\
\hline $\begin{array}{l}\text { 2.3b Los trabajadores conocen el propósito de la } \\
\text { compañía }\end{array}$ & 1 & 2 & 3 & 4 & 5 \\
\hline $\begin{array}{l}\text { 2.3c Los trabajadores se esfuerzan por mantener } \\
\text { los principios y valores de la compañía }\end{array}$ & 1 & 2 & 3 & 4 & 5 \\
\hline $\begin{array}{l}\text { 2.3d Se promueve una cultura de seguridad entre } \\
\text { los trabajadores }\end{array}$ & 1 & 2 & 3 & 4 & 5 \\
\hline $\begin{array}{l}\text { 2.3e Se realizan inspecciones de seguridad con } \\
\text { regularidad }\end{array}$ & 1 & 2 & 3 & 4 & 5 \\
\hline 2.3f Se está a favor del cambio & 1 & 2 & 3 & 4 & 5 \\
\hline $\begin{array}{l}\text { 2.3g La división del trabajo realmente ayuda a } \\
\text { cumplir las metas }\end{array}$ & 1 & 2 & 3 & 4 & 5 \\
\hline $\begin{array}{l}\text { 2.4a Los horarios de trabajo son cómodos y se } \\
\text { mantienen estables }\end{array}$ & 1 & 2 & 3 & 4 & 5 \\
\hline $\begin{array}{l}\text { 2.4b Se informa con anticipación a los } \\
\text { trabajadores sobre aquellos trabajos que van a }\end{array}$ & 1 & 2 & & 4 & 5 \\
\hline $\begin{array}{l}2.4 c \text { Se les permite a los trabajadores tomar sus } \\
\text { requer tiempo extra }\end{array}$ & 1 & 2 & 3 & 4 & 5 \\
\hline $\begin{array}{l}\text { propias decisiones sobre cómo programar su } \\
\text { trabajo }\end{array}$ & 1 & 2 & 3 & 4 & 5 \\
\hline $\begin{array}{l}2.5 a \text { Se permiten las relaciones sociales entre los } \\
\text { trabajadores dentro de la planta }\end{array}$ & 1 & 2 & 3 & 4 & 5 \\
\hline $\begin{array}{l}2.5 \mathrm{~b} \text { Los trabajadores mantienen relaciones } \\
\text { profesionales cordiales }\end{array}$ & 1 & 2 & 3 & 4 & 5 \\
\hline $\begin{array}{l}2.5 \mathrm{c} \text { Se comparte la información entre los } \\
\text { trabajadores }\end{array}$ & 1 & 2 & 3 & 4 & 5 \\
\hline $\begin{array}{l}2.5 \mathrm{~d} \text { Los trabajadores pueden reunirse para } \\
\text { convivir/platicar de cualquier tema }\end{array}$ & 1 & 2 & 3 & 4 & 5 \\
\hline $\begin{array}{l}\text { 2.5e Los superiores se preocupan por el bienestar } \\
\text { de las personas que trabajan para ellos }\end{array}$ & 1 & 2 & 3 & 4 & 5 \\
\hline $\begin{array}{l}2.5 f \text { Los trabajadores interactúan con personas } \\
\text { ajenas a la organización }\end{array}$ & 1 & 2 & 3 & 4 & 5 \\
\hline $\begin{array}{l}\text { 2.6a Las tareas se supervisan y administran para } \\
\text { mejorar el desempeño de los trabajadores }\end{array}$ & 1 & 2 & 3 & 4 & 5 \\
\hline $\begin{array}{l}\text { 2.6b Los supervisores brindan atención y apoyo a } \\
\text { los trabajadores de manera continua }\end{array}$ & 1 & 2 & 3 & 4 & 5 \\
\hline $\begin{array}{l}\text { 2.7a Se realizan evaluaciones del desempeño de } \\
\text { los trabajadores de manera periódica }\end{array}$ & 1 & 2 & 3 & 4 & 5 \\
\hline
\end{tabular}




\begin{tabular}{|c|c|c|c|c|c|}
\hline \multicolumn{6}{|c|}{ Evaluación de condiciones admintsativas de la organización } \\
\hline \multicolumn{6}{|c|}{ 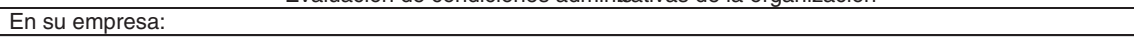 } \\
\hline & $\begin{array}{l}\text { Totalmente } \\
\quad \text { en } \\
\text { desacuerdo }\end{array}$ & $\begin{array}{c}\text { Muy en } \\
\text { desacuerdo }\end{array}$ & $\begin{array}{c}\text { Ni de } \\
\text { acuerdo ni } \\
\text { en } \\
\text { desacuerdo }\end{array}$ & $\begin{array}{l}\text { Muy de } \\
\text { acuerdo }\end{array}$ & $\begin{array}{l}\text { Totalmente } \\
\quad \text { de } \\
\text { acuerdo }\end{array}$ \\
\hline $\begin{array}{l}\text { 2.7b Se motiva a los trabajadores a dar su mejor } \\
\text { esfuerzo }\end{array}$ & 1 & 2 & ( & 4 & 5 \\
\hline $\begin{array}{l}\text { 2.7c El salario de los trabajadores es } \\
\text { conmensurado con el trabajo que ellos realizan }\end{array}$ & 1 & 2 & 3 & 4 & 5 \\
\hline $\begin{array}{l}2.7 \mathrm{~d} \text { Todas las tareas que se realizan están } \\
\text { asociadas con incentivos }\end{array}$ & 1 & 2 & 3 & 4 & 5 \\
\hline \multicolumn{6}{|c|}{ Evaluación de aspectos tecnológicos } \\
\hline \multicolumn{6}{|l|}{ En su empresa: } \\
\hline & $\begin{array}{l}\text { Totalmente } \\
\quad \text { en } \\
\text { desacuerdo }\end{array}$ & $\begin{array}{c}\text { Muy en } \\
\text { desacuerdo }\end{array}$ & $\begin{array}{c}\text { Ni de } \\
\text { acuerdo ni } \\
\text { en } \\
\text { desacuerdo }\end{array}$ & $\begin{array}{l}\text { Muy de } \\
\text { acuerdo }\end{array}$ & $\begin{array}{l}\text { Totalmente } \\
\quad \text { de } \\
\text { acuerdo }\end{array}$ \\
\hline $\begin{array}{l}\text { 3.1a Se toman en cuenta factores humanos y } \\
\text { ergonómicos al momento de adquirir tecnología de } \\
\text { información }\end{array}$ & 1 & 2 & (2) & 4 & 5 \\
\hline $\begin{array}{l}\text { 3.1b El trabajo requiere del uso de diferentes } \\
\text { tecnologías de información }\end{array}$ & 1 & 2 & 3 & 4 & 5 \\
\hline $\begin{array}{l}\text { 3.1c El trabajo realizado con las tecnologías de } \\
\text { información se lleva a cabo en un ambiente libre de } \\
\text { riesgos }\end{array}$ & 1 & 2 & 3 & 4 & 5 \\
\hline $\begin{array}{l}\text { 3.1d Los trabajadores reciben información sobre la } \\
\text { tecnología que utilizan }\end{array}$ & 1 & 2 & 3 & 4 & 5 \\
\hline $\begin{array}{l}\text { 3.1e Los trabajadoresmuestran actitudes positivas } \\
\text { hacia la tecnología que utilizan }\end{array}$ & 1 & 2 & 3 & 4 & 5 \\
\hline $\begin{array}{l}\text { 3.1f La tecnología tiene un impacto positivo en el } \\
\text { desempeño y bienestar de los trabajadores }\end{array}$ & 1 & 2 & 3 & 4 & 5 \\
\hline $\begin{array}{l}\text { 3.1g El uso y la adaptación de las tecnologías de la } \\
\text { información requiere poco tiempo }\end{array}$ & $\mathrm{a}$ & 2 & 3 & 4 & 5 \\
\hline $\begin{array}{l}\text { 3.2a Se utilizan diferentes tecnologías de } \\
\text { manufactura }\end{array}$ & 1 & 2 & 3 & 4 & 5 \\
\hline $\begin{array}{l}\text { 3.2b Las tecnologías de manufactura se adquieren } \\
\text { con base en la consideración de factores humanos y } \\
\text { ergonómicos }\end{array}$ & $y$ & 2 & 3 & 4 & 5 \\
\hline $\begin{array}{l}\text { 3.2c El uso de tecnología de manufactura se lleva } \\
\text { a cabo en un ambiente libre de riesgos }\end{array}$ & 1 & 2 & 3 & 4 & 5 \\
\hline $\begin{array}{l}\text { 3.2d El uso o la adaptación de las tecnologías de } \\
\text { manufactura requiere poco tiempo }\end{array}$ & 1 & 2 & 3 & 4 & 5 \\
\hline $\begin{array}{l}\text { 3.3a Se toman en cuenta factores humanos y } \\
\text { ergonómicos al momento de adquirir tecnología y } \\
\text { herramientas de manufactura }\end{array}$ & 1 & 2 & 3 & 4 & 5 \\
\hline $\begin{array}{l}\text { 3.3b Las tareas realizadas con herramientas } \\
\text { manuales son seguras para el trabajador }\end{array}$ & 1 & 2 & 3 & 4 & 5 \\
\hline
\end{tabular}

\begin{tabular}{lccccc}
\hline \multicolumn{7}{c}{ Evaluación de aspectos relacionados con las tareas } \\
\hline En su empresa: & $\begin{array}{c}\text { Totalmente } \\
\text { en } \\
\text { desacuerdo }\end{array}$ & $\begin{array}{c}\text { Muy en } \\
\text { desacuerdo }\end{array}$ & $\begin{array}{c}\text { Ni de } \\
\text { acuerdo ni } \\
\text { en } \\
\text { desacuerdo }\end{array}$ & $\begin{array}{c}\text { Muy de } \\
\text { acuerdo }\end{array}$ & $\begin{array}{c}\text { Totalmente } \\
\text { de } \\
\text { acuerdo }\end{array}$ \\
\hline $\begin{array}{l}\text { 4.1a Los trabajadores realizan diferentes tareas } \\
\text { manteniendo un buen desempeño }\end{array}$ & 1 & 2 & 3 & 4 & 5 \\
$\begin{array}{l}\text { 4.1b Se promueve el aprendizaje de diferentes } \\
\text { tareas por parte de los trabajadores }\end{array}$ & 1 & 2 & 3 & 4 & 5 \\
$\begin{array}{l}\text { 4.1c Se realiza rotación de puestos por los } \\
\text { trabajadores durante la misma jornada de trabajo }\end{array}$ & 1 & 2 & 3 & 4 & 5 \\
\hline
\end{tabular}




\begin{tabular}{|c|c|c|c|c|c|}
\hline \multicolumn{6}{|c|}{ Evaluación de aspectos relacionados con las tareas } \\
\hline \multicolumn{6}{|l|}{ En su empresa: } \\
\hline & $\begin{array}{l}\text { Totalmente } \\
\text { en } \\
\text { desacuerdo }\end{array}$ & $\begin{array}{c}\text { Muy en } \\
\text { desacuerdo }\end{array}$ & $\begin{array}{c}\text { Ni de } \\
\text { acuerdo ni } \\
\text { en } \\
\text { desacuerdo } \\
\end{array}$ & $\begin{array}{l}\text { Muy de } \\
\text { acuerdo }\end{array}$ & $\begin{array}{l}\text { Totalmente } \\
\quad \text { de } \\
\text { acuerdo }\end{array}$ \\
\hline $\begin{array}{l}\text { 4.2a Se aprovechan las habilidades actuales de los } \\
\text { trabajadores }\end{array}$ & 1 & 2 & 3 & 4 & 5 \\
\hline $\begin{array}{l}\text { 4.2b Se promueve la adquisición de nuevas } \\
\text { habilidades de los trabajadores mediante la } \\
\text { ejecución de tareas que representan un desafío para } \\
\text { ellos }\end{array}$ & ra & 2 & 3 & 4 & 5 \\
\hline $\begin{array}{l}\text { 4.3a Se les permite a los trabajadores tomar sus } \\
\text { propias decisiones sobre la agenda de trabajo }\end{array}$ & 1 & 2 & 3 & 4 & 5 \\
\hline $\begin{array}{l}4.3 \mathrm{~b} \text { Se les permite a los trabajadores decidir qué } \\
\text { métodos utilizar para realizar una tarea }\end{array}$ & 1 & 2 & 3 & 4 & 5 \\
\hline $\begin{array}{l}\text { 4.3c Los trabajadores deciden el orden en el cual } \\
\text { realizan las tareas }\end{array}$ & 1 & 2 & 3 & 4 & 5 \\
\hline $\begin{array}{l}\text { 4.3d Se les permite a lostrabajadores utilizar su } \\
\text { iniciativa personal }\end{array}$ & 1 & 2 & 3 & 4 & 5 \\
\hline $\begin{array}{l}\text { 4.3e Se les permite a los trabajadores participar en } \\
\text { propuestas de mejora }\end{array}$ & 1 & 2 & 3 & 4 & 5 \\
\hline $\begin{array}{l}\text { 4.4a Las tareas están diseñadas para que los } \\
\text { trabajadores realicen una sola tarea a la vez }\end{array}$ & 1 & 2 & 3 & 4 & 5 \\
\hline $\begin{array}{l}\text { 4.4b Las tareas que se realizan son simples y sin } \\
\text { complicaciones }\end{array}$ & 1 & 2 & 3 & 4 & 5 \\
\hline $\begin{array}{l}\text { 4.4c Los trabajadores procesan/monitorean poca } \\
\text { cantidad de información durante la ejecución de } \\
\text { sus tareas }\end{array}$ & 1 & 2 & 3 & 4 & 5 \\
\hline $\begin{array}{l}\text { 4.4d Las tareas requieren la resolución de } \\
\text { problemas cuya respuesta correcta es obvia }\end{array}$ & 1 & 2 & 3 & 4 & 5 \\
\hline $\begin{array}{l}\text { 4.4e Las tareas requieren de la creatividad de los } \\
\text { trabajadores }\end{array}$ & 1 & 2 & 3 & 4 & 5 \\
\hline $\begin{array}{l}4.4 f \text { Se evalúan las tareas de manera periódica } \\
\text { sobre la carga de trabajo }\end{array}$ & 1 & 2 & 3 & 4 & 5 \\
\hline
\end{tabular}

\begin{tabular}{|c|c|c|c|c|c|}
\hline \multicolumn{6}{|c|}{ Evaluación de aspectos ambientales } \\
\hline \multicolumn{6}{|l|}{ En su empresa: } \\
\hline & $\begin{array}{c}\text { Totalmente } \\
\text { en } \\
\text { desacuerdo }\end{array}$ & $\begin{array}{l}\text { Muy en } \\
\text { desacuerdo }\end{array}$ & $\begin{array}{c}\text { Ni de } \\
\text { acuerdo ni } \\
\text { en } \\
\text { desacuerdo }\end{array}$ & $\begin{array}{l}\text { Muy de } \\
\text { acuerdo }\end{array}$ & $\begin{array}{l}\text { Totalmente } \\
\quad \text { de } \\
\text { acuerdo }\end{array}$ \\
\hline $\begin{array}{l}\text { 5.1a La distribución de las instalaciones, equipos, } \\
\text { etc. permiten movimientos y posturas cómodas a } \\
\text { los trabajadores }\end{array}$ & 1 & 2 & 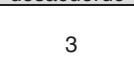 & 4 & 5 \\
\hline $\begin{array}{l}\text { 5.1b La distribución de las instalaciones, equipos, } \\
\text { etc. permiten que los trabajadores realicen } \\
\text { traslados frecuentes a corta distancia }\end{array}$ & 1 & 2 & 3 & 4 & 5 \\
\hline $\begin{array}{l}\text { 5.1c La distribución de las instalaciones, equipos, } \\
\text { etc. permiten mejores transportes de material }\end{array}$ & 1 & 2 & 3 & 4 & 5 \\
\hline $\begin{array}{l}\text { 5.1d La distribución de las instalaciones, equipos, } \\
\text { etc. permiten monitorear tareas a distancia }\end{array}$ & 1 & 2 & 3 & 4 & 5 \\
\hline $\begin{array}{l}\text { 5.1e La distribución de las instalaciones, equipos, } \\
\text { etc. facilitan las diferentes tipos de comunicación } \\
\text { (visual, auditiva) entre los trabajadores }\end{array}$ & 1 & 2 & 3 & 4 & 5 \\
\hline $\begin{array}{l}\text { 5.2a Las tareas se realizen un ambiente libre de } \\
\text { ruido excesivo }\end{array}$ & 1 & 2 & 3 & 4 & 5 \\
\hline $\begin{array}{l}5.2 \mathrm{~b} \text { Los trabajadores están aislados de las } \\
\text { tareas/maquinaria que emite ruido excesivo }\end{array}$ & 1 & 2 & 3 & 4 & 5 \\
\hline $\begin{array}{l}\text { 5.2c Se realizan evaluaciones periódicas del nivel } \\
\text { de ruido }\end{array}$ & l & 2 & 3 & 4 & 5 \\
\hline
\end{tabular}




\begin{tabular}{|c|c|c|c|c|c|}
\hline \multicolumn{6}{|c|}{ Evaluación de aspectos ambientales } \\
\hline \multicolumn{6}{|l|}{ En su empresa: } \\
\hline & $\begin{array}{l}\text { Totalmente } \\
\text { en } \\
\text { desacuerdo }\end{array}$ & $\begin{array}{c}\text { Muy en } \\
\text { desacuerdo }\end{array}$ & $\begin{array}{c}\text { Ni de } \\
\text { acuerdo ni } \\
\text { en } \\
\text { desacuerdo } \\
\end{array}$ & $\begin{array}{r}\text { Muy de } \\
\text { acuerdo }\end{array}$ & $\begin{array}{c}\text { Totalmente } \\
\text { de } \\
\text { acuerdo }\end{array}$ \\
\hline $\begin{array}{l}\text { 5.3a Las tareas se realizan con un nivel de } \\
\text { iluminación seguro y eficiente }\end{array}$ & 1 & 2 & 3 & 4 & 5 \\
\hline $\begin{array}{l}\text { 5.3b Se realizan evaluaciones periódicas del nivel } \\
\text { de iluminación }\end{array}$ & 1 & 2 & 3 & 4 & 5 \\
\hline $\begin{array}{l}\text { 5.4a Las tareas se realizan en un ambiente cómodo } \\
\text { en términos de condiciones climáticas }\end{array}$ & do & 2 & 3 & 4 & 5 \\
\hline $\begin{array}{l}\text { 5.4b Los trabajadores están aislados de las } \\
\text { tareas/maquinaria que emiten altas temperaturas }\end{array}$ & 1 & 2 & 3 & 4 & 5 \\
\hline $\begin{array}{l}\text { 5.4c Las tareas se realizan en un ambiente de aire } \\
\text { limpio (sin químicos, gases, etc.) }\end{array}$ & 1 & 2 & 3 & 4 & 5 \\
\hline $\begin{array}{l}\text { 5.4d Se realizan evaluaciones periódicas de la } \\
\text { temperatura, humedad y calidad del aire }\end{array}$ & 1 & 2 & 3 & 4 & 5 \\
\hline $\begin{array}{l}5.4 \mathrm{e} \text { Los trabajadores utilizan equipo de } \\
\text { protección personal contra ruido, temperatura, } \\
\text { humedad, químicos en las tareas que así lo } \\
\text { requieren }\end{array}$ & 1 & 2 & 3 & 4 & 5 \\
\hline $\begin{array}{l}\text { 5.5a En aquellas estaciones de trabajo que se } \\
\text { realizan en postura sedente (sentado), las sillas o } \\
\text { bancos son cómodos y presentan un buen soporte. } \\
\text { Así mismo, permiten el cambio de postura, para } \\
\text { trabajar de pie. }\end{array}$ & . & 2 & 3 & 4 & 5 \\
\hline $\begin{array}{l}5.5 \mathrm{~b} \text { El diseño de las estaciones de trabajo permite } \\
\text { la ajustabilidad al trabajador }\end{array}$ & 1 & 2 & 3 & 4 & 5 \\
\hline $\begin{array}{l}\text { 5.5c Los trabajadores realizan pocos movimientos } \\
\text { de alcance durante la ejecución de sus tareas }\end{array}$ & 1 & 2 & 3 & 4 & 5 \\
\hline $\begin{array}{l}\text { 5.5d Se realizan evaluaciones periódicas del } \\
\text { diseño de las estaciones de trabajo }\end{array}$ & 1 & 2 & 3 & 4 & 5 \\
\hline
\end{tabular}

\section{Referencias}

Åkerstedt, T., Fredlund, P., Gillberg, M. y Jansson, B. (2002). Work load and work hours in relation to disturbed sleep and fatigue in a large representative sample. Journal of Psychosomatic Research, 53(1), 585-588.

Anderson, D. J., Binder, M. y Krause, K. (2002). The motherhood wage penalty revisited: Experience, heterogeneity, work effort, and work-schedule flexibility. Indus. \& Lab. Rel. Rev., 56, 273.

Axtell, C. M., Holman, D. J., Unsworth, K. L., Wall, T. D., Waterson, P. E. y Harrington, E. (2000). Shopfloor innovation: Facilitating the suggestion and implementation of ideas. Journal of Occupational and Organizational Psychology, 73(3), 265-285.

Balbinotti, G. y Paupitz, A. (2015). Internationally management of socio-technical projects-A case study in a vehicle assembly. Procedia Manufacturing, 3, 6345-6352.

Beckers, D. G. J., van der Linden, D., Smulders, P. G. W., Kompier, M. A. J., van Veldhoven, M. J. P. M. y van Yperen, N. W. (2004). Working overtime hours: Relations with fatigue, work motivation, and the quality of work. Journal of Occupational and Environmental Medicine, 46(12), 1282-1289.

Bolis, I. y Sznelwar, L. I. (2015). A case study of the implementation of an ergonomics improvement committee in a Brazilian hospital-Challenges and benefits. Applied ergonomics.

Cammann, C., Fichman, M., Jenkins, D. y Klesh, J. (1979). The Michigan organizational assessment questionnaire [unpublished manuscript]. Ann Arbor: University of Michigan.

Carayon, P. y Hoonakker, P. L. T. (2004). Macroergonomic Organizational Questionnaire Survey (MOQS). En N. A. Stanton, A. Hedge, K. Brookhuis, E. Salas, y H. W. Hendrick (Eds.), Handbook of human factors and ergonomic methods. Boca Raton, Florida: CRC Press, 76-71.

Carayon, P., Hundt, A. S., Karsh, B. T., Gurses, A. P., Alvarado, C. J., Smith, M., et al. (2006). Work system design for patient safety: the SEIPS model. Quality and Safety in Health Care, 15(Suppl 1), i50-i58.

Celik, M., Kahraman, C., Cebi, S. y Er, I. D. (2009). Fuzzy axiomatic design-based performance evaluation model for docking facilities in shipbuilding industry: The case of Turkish shipyards. Expert Systems with Applications, 36(1), 599-615. 
Choudhry, R. M., Fang, D. y Mohamed, S. (2007). The nature of safety culture: A survey of the state-of-the-art. Safety Science, 45(10), 993-1012.

Coluci, M. Z. O., Alexandre, N. M. C. y Rosecrance, J. (2009). Reliability and validity of an ergonomics-related Job Factors Questionnaire. International Journal of Industrial Ergonomics, 39(6), 995-1001.

Cronbach, L. J. (1951). Coefficient alpha and the internal structure of tests. Psychometrika, 16(3), $297-334$.

Detert, J. R. y Burris, E. R. (2007). Leadership behavior and employee voice: Is the door really open? Academy of Management Journal, 50(4), 869-884.

Di Pietro, L., Pantano, E. y di Virgilio, F. (2014). Frontline employees' attitudes towards self-service technologies: Threats or opportunity for job performance? Journal of Retailing and Consumer Services, 21(5), 844-850, doi: http://dx.doi.org/10.1016/j.jretconser.2014.02.014

Fornell, C. y Larcker, D. F. (1981). Evaluating structural equation models with unobservable variables and measurement error. Journal of Marketing Research, 39-50.

García, J. L., Maldonado, A. A., Alvarado, A. y Rivera, D. G. (2014). Human critical success factors for kaizen and its impacts in industrial performance. The International Journal of Advanced Manufacturing Technology, 70(9-12), 2187-2198.

García-Acosta, G. y Lange-Morales, K. (2008). Macroergonomic study of food sector company distribution centres. Applied Ergonomics, 39(4), 439-449, doi: http://dx.doi.org/10.1016/j.apergo.2008.02.004

Giunta, A., Nifo, A. y Scalera, D. (2012). Subcontracting in Italian industry: Labour division, firm growth and the north-south divide. Regional Studies, 46(8), 1067-1083.

Glover, W. J., Farris, J. A., van Aken, E. M. y Doolen, T. L. (2011). Critical success factors for the sustainability of Kaizen event human resource outcomes: An empirical study. International Journal of Production Economics, 132(2), 197-213.

Golshan, N. y Tafazoli, D. (2014). Technology-enhanced language learning tools in Iranian EFL context: Frequencies, attitudes and challenges. Procedia-Social and Behavioral Sciences, 136(0), 114-118, doi: http://dx.doi.org/10.1016/j.sbspro.2014.05.299

Hair, J. F., Tatham, R. L., Anderson, R. E. y Black, W. C. (2006). . Multivariate data analysis (Vol. 6) Upper Saddle River, NJ: Pearson Prentice Hall.

Häusser, J. A., Mojzisch, A., Niesel, M. y Schulz-Hardt, S. (2010). Ten years on: A review of recent research on the Job demand-control (-support) model and psychological well-being. Work \& Stress, 24(1), 1-35.

Hildebrandt, V. H., Bongers, P. M., van Dijk, F. J. H., Kemper, H. C. G. y Dul, J. (2001). Dutch Musculoskeletal Questionnaire: Description and basic qualities. Ergonomics, 44(12), 1038-1055.

Horst, D. J., Broday, E. E., Bondarik, R. y de Paula Xavier, A. A. (2015). Open or closed? Macroergonomics approaching socio-technical systems productivity: An overview of the conceptual framework. Social and Basic Sciences Research Review, 3(2), 98-115.

Humphrey, S. E., Nahrgang, J. D. y Morgeson, F. P. (2007). Integrating motivational, social, and contextual work design features: A meta-analytic summary and theoretical extension of the work design literature. Journal of Applied Psychology, 92(5), 1332.

Instituto-Nacional-de-Estadística-Geografía-e-Informática-(INEGI). (2015). Estadística Integral de Programa de la Industria Manufacturera, Maquiladora y Servicios de Exportación (IMMEX).

Jung, M.-C. y Hallbeck, M. S. (2005). Ergonomic redesign and evaluation of a clamping tool handle. Applied Ergonomics, 36(5), 619-624, doi: http://dx.doi.org/10.1016/j.apergo.2005.01.016

Kamboj, S. K., Oldfield, L., Loewenberger, A., Das, R. K., Bisby, J. y Brewin, C. R. (2014). Voluntary and involuntary emotional memory following an analogue traumatic stressor: The differential effects of communality in men and women. Journal of Behavior Therapy and Experimental Psychiatry, 45(4), 421-426, doi: http://dx.doi.org/10.1016/j.jbtep.2014.05.001

Karasek, R. (1985). Job content questionnaire and user's guide. Lowell: University of Massachusetts.

Karasek, R., Brisson, C., Kawakami, N., Houtman, I., Bongers, P. y Amick, B. (1998). The Job Content Questionnaire (JCQ): An instrument for internationally comparative assessments of psychosocial job characteristics. Journal of Occupational Health Psychology, 3(4), 322.

Kleiner, M. B. (1998). Macroergonomic analysis of formalization in a dynamic work system. Applied Ergonomics, 29(4), 255-259.

Kleiner, M. B. (2006). Macroergonomics: analysis and design of work systems. Applied Ergonomics, 37(1), 81-89.

Kleiner, M. B. (2008). Macroergonomics: Work system analysis and design. Human Factors: The Journal of the Human Factors and Ergonomics Society, 50(3), 461-467.

Li, Q. (2013). A novel Likert scale based on fuzzy sets theory. Expert Systems with Applications, 40(5), 1609-1618, doi: http://dx.doi.org/10.1016/j.eswa.2012.09.015 
Likert, R. (1932). A technique for the measurement of attitudes. Archives of psychology.

Lipscomb, J. A., Trinkoff, A. M., Geiger-Brown, J. y Brady, B. (2002). Work-schedule characteristics and reported musculoskeletal disorders of registered nurses. Scandinavian journal of work, environment y health, 394-401.

Maldonado, A., García, J. L., Alvarado, A. y Balderrama, C. O. (2013). A hierarchical fuzzy axiomatic design methodology for ergonomic compatibility evaluation of advanced manufacturing technology. The International Journal of Advanced Manufacturing Technology, 66(1-4), 171-186.

Maldonado, A., Sánchez, J., Noriega, S., Díaz, J. J., García, J. L., y Vidal, L. (2009). A hierarchical fuzzy axiomatic design survey for ergonomic compatibility evaluation of advanced manufacturing technology -AMT. Paper presented at the Proceedings of the XXIst. Annual International Conference, International Society for Occupational Ergonomics and Safety.

Mital, A. y Pennathur, A. (2004). Advanced technologies and humans in manufacturing workplaces: An interdependent relationship. International Journal of Industrial Ergonomics, 33(4), 295-313.

Mital, A., Pennathur, A., Huston, R. L., Thompson, D., Pittman, M., Markle, G., et al. (1999). The need for worker training in advanced manufacturing technology (AMT) environments: A white paper. International Journal of Industrial Ergonomics, 24(2), 173-184.

Morel, G., Amalberti, R. y Chauvin, C. (2009). How good micro/macro ergonomics may improve resilience, but not necessarily safety. Safety Science, 47(2), 285-294, doi: http://dx.doi.org/10.1016/j.ssci.2008.03.002

Morgeson, F.P., y Humphrey, S.E. (2003). Work Design Questionnaire (WDQ): Developing and validating a comprehensive measure of work design. Paper presented at the 62nd Annual Meeting of the Academy of Management, Seattle, WA.

Morgeson, F. P. y Humphrey, S. E. (2006). The Work Design Questionnaire (WDQ): Developing and validating a comprehensive measure for assessing job design and the nature of work. Journal of Applied Psychology, 91(6), 1321.

Morosan, C. y DeFranco, A. (2014). When tradition meets the new technology: An examination of the antecedents of attitudes and intentions to use mobile devices in private clubs. International Journal of Hospitality Management, 42, 126-136, doi: http://dx.doi.org/10.1016/j.ijhm.2014.06.009

Nakata, A., Haratani, T., Takahashi, M., Kawakami, N., Arito, H., Kobayashi, F., et al. (2004). Job stress, social support, and prevalence of insomnia in a population of Japanese daytime workers. Social Science \& Medicine, 59(8), 1719-1730.

Nunnally, J. C. (1978). Psychometric theory: New York: McGraw-Hill.

Nunnally, J.C., y Bernstein, H. (2005). Psychometric theory. México, DF: M.H. Interamericana Ed (en español).

Oldham, G. R. y Cummings, A. (1996). Employee creativity: Personal and contextual factors at work. Academy of Management Journal, 39(3), 607-634.

Oyewole, S. A., Haight, J. M. y Freivalds, A. (2010). The ergonomic design of classroom furniture/computer work station for first graders in the elementary school. International Journal of Industrial Ergonomics, 40(4), 437-447.

Öztürk, N. y Esin, M. N. (2011). Investigation of musculoskeletal symptoms and ergonomic risk factors among female sewing machine operators in Turkey. International Journal of Industrial Ergonomics, 41(6), 585-591.

Pavlovic-Veselinovic, S., Hedge, A. y Veselinovic, M. (2016). An ergonomic expert system for risk assessment of work-related musculo-skeletal disorders. International Journal of Industrial Ergonomics, 53, 130-139, doi: http://dx.doi.org/10.1016/j.ergon.2015.11.008

Pennathur, A. y Mital, A. (2003). Worker mobility and training in advanced manufacturing. International Journal of Industrial Ergonomics, 32(6), 363-388.

Preziosi, R. C. (1980). Organisational Diagnosis Questionnaire (ODQ). The 1980 annual handbook for group facilitators, $112-120$.

Realyvásquez, A., Maldonado-Macías, A. A., García-Alcaraz, J. L. y Blanco-Fernández, J. (2015). Effects of organizational macroergonomic compatibility elements over manufacturing systems' performance. Procedia Manufacturing, 3, 5715-5722.

Reinhold, K. y Tint, P. (2015). Lighting of workplaces and health risks. Elektronika ir Elektrotechnika, 90(2), 11-14.

Roberts, G. E. (2003). Employee performance appraisal system participation: A technique that works. Public Personnel Management, 32(1), 89-98.

Robertsona, M.M., Huanga, Y.-H., y Leea, J. (2015). Effects of a macroergonomics intervention: Examining the relationship of environmental control. Paper presented at the Proceedings 19th Triennial Congress of the IEA.

Schulze, R., Hilger, A.-L. y Engelberg, P. M. (2015). Factor analysis and latent variable models in personality psychology. En J. D. Wright (Ed.), International Encyclopedia of the Social y Behavioral Sciences (2nd ed., Vol. 6, pp. 703-708). Oxford: Elsevier.

Seim, R. y Broberg, O. (2010). Participatory workspace design: A new approach for ergonomists? International Journal of Industrial Ergonomics, 40(1), 25-33. 
Sen, R. N. y Sahu, S. (1996). Ergonomic evaluation of a multipurpose shovel-cum-hoe for manual material handling. International Journal of Industrial Ergonomics, 17(1), 53-58, doi: http://dx.doi.org/10.1016/0169-8141(94)00105-7

Thompson, C. A. y Prottas, D. J. (2006). Relationships among organizational family support, job autonomy, perceived control, and employee well-being. Journal of Occupational Health Psychology, 11(1), 100.

Trinkoff, A. M., Le, R., Geiger-Brown, J. y Lipscomb, J. (2007). Work schedule, needle use, and needlestick injuries among registered nurses. Work, 28(2), 156-164.

Valdez, R., y Zayas-Cabán, T. (2015). Developing hybrid macroergonomic methodologies: a systems foundation for consumer health IT design. Paper presented at the Proceedings 19th Triennial Congress of the IEA.

Van der Doef, M. y Maes, S. (1999). The job demand-control (-support) model and psychological well-being: A review of 20 years of empirical research. Work \& Stress, 13(2), 87-114.

Wang, H., Liu, Q. y Tu, Y. (2005). Interpretation of partial least-squares regression models with VARIMAX rotation. Computational Statistics \& Data Analysis, 48(1), 207-219, doi: http://dx.doi.org/10.1016/j.csda.2003.12.005

Wilson, J. R. (2000). Fundamentals of ergonomics in theory and practice. Applied Ergonomics, 31(6), 557-567.

Wilson, J. R. y Sharples, S. (2015). Evaluation of human work. Boca Raton, Florida: CRC Press.

Yang, Y. y Rivera, A. J. (2015). An observational study of hands-free communication devices mediated interruption dynamics in a nursing work system. Health Policy and Technology, 4(4), 378-386.

Yilmaz, O. y Bayraktar, D. M. (2014). Teachers' attitudes towards the use of educational technologies and their individual innovativeness categories. Procedia-Social and Behavioral Sciences, 116, 3458-3461, doi: http://dx.doi.org/10.1016/j.sbspro.2014.01.783

You, H., Kumar, A., Young, R., Veluswamy, P. y Malzahn, D. E. (2005). An ergonomic evaluation of manual Cleco plier designs: Effects of rubber grip, spring recoil, and worksurface angle. Applied Ergonomics, 36(5), 575-583, doi: http://dx.doi.org/10.1016/j.apergo.2005.01.014

Zhao, D., McCoy, A. P., Kleiner, B. M., Smith-Jackson, T. L. y Liu, G. (2015). Sociotechnical systems of fatal electrical injuries in the construction industry. Journal of Construction Engineering and Management, 04015056.

Zhu, D. H. y Chang, Y. P. (2014). Investigating consumer attitude and intention toward free trials of technology-based services. Computers in Human Behavior, 30, 328-334, doi: http://dx.doi.org/10.1016/j.chb.2013.09.008

Zink, K. J. (2014). Designing sustainable work systems: The need for a systems approach. Applied Ergonomics, 45(1), 126-132. 